\title{
Exosomal IncRNA UCA1 Derived From Pancreatic Stellate Cells Promotes Gemcitabine Resistance in Pancreatic Cancer via the SOCS3/EZH2 Axis
}

Yuan Chi, He Xin and Zhaoyu Liu*

Department of Radiology, Shengjing Hospital of China Medical University, Shenyang, China

OPEN ACCESS

Edited by:

Zexian Liu,

Sun Yat-sen University Cancer Center

(SYSUCC), China

Reviewed by:

Jun Li,

St. Jude Children's Research Hospital, United States

Allen Thayakumar Basanthakumar, Dana-Farber Cancer Institute, United States

${ }^{*}$ Correspondence:

Zhaoyu Liu

liuzy@sj-hospital.org

Specialty section: This article was submitted to Gastrointestinal Cancers, a section of the journal

Frontiers in Oncology

Received: 23 February 2021 Accepted: 31 August 2021

Published: 19 November 2021

Citation:

Chi Y, Xin H and Liu Z (2021) Exosomal InCRNA UCA1 Derived From

Pancreatic Stellate Cells Promotes Gemcitabine Resistance in Pancreatic Cancer via the SOCS3/EZH2 Axis.

Front. Oncol. 11:671082.

doi: 10.3389/fonc.2021.671082
Objective: Pancreatic cancer is associated with poor prognosis and dismal survival rates. This study aims to investigate roles of IncRNA UCA1-loaded exosomes secreted by pancreatic stellate cells (PSCs) in Gemcitabine (Gem) resistance of pancreatic cancer under hypoxia, which involves the methylation of SOCS3 and EZH2 recruitment.

Methods: The exosomes were isolated from PSCs and hypoxic PSCs (HPSCs), and cocultured with pancreatic cancer cells transduced with manipulated IncRNA UCA1, EZH2, and SOCS3. The interaction among IncRNA UCA1, EZH2, and SOCS3 was characterized by RIP and ChIP assays. Next, MTT assay, flow cytometry and TUNEL staining and Transwell assay were used to detect cell viability, apoptosis, invasion, and migration. Gem-resistant pancreatic cancer cell line (GemMIA-R3) was established, which was applied in a mouse xenograft model of pancreatic cancer, with MTT assay to determine Gem sensitivity.

Results: LncRNA UCA1 was highly expressed, while SOCS3 was poorly expressed in pancreatic cancer tissues. Hypoxia induced activation of PSCs and promoted release of exosomes. LncRNA UCA1 delivered by hypoxic PSC-derived exosomes (HPSC-EXO) regulated histone methylation level in SOCS3 gene region through recruitment of EZH2. In vitro and in vivo experimental results confirmed that IncRNA UCA1-loaded HPSC-EXO promoted malignant phenotypes, inhibited apoptosis, and promoted Gem resistance of pancreatic cancer cells as well as tumorigenesis in mice.

Conclusion: Under hypoxic conditions, exosomes secreted by hypoxia-induced PSCs deliver IncRNA UCA1 into pancreatic cancer cells, where IncRNA UCA1 recruits EZH2 and regulates histone methylation level in SOCS3 gene region, thereby augmenting pancreatic cancer resistance to Gem.

Keywords: pancreatic cancer, hypoxia, pancreatic stellate cells, exosomes, IncRNA UCA1, gemcitabine resistance 


\section{INTRODUCTION}

Pancreatic cancer has a poor prognosis and accounts for about $3 \%$ of new cancer cases each year (1). Pancreatic stellate cells (PSCs) are the main cell components of pancreatic cancer, which produce large number of extracellular matrix components (2). Increasing evidence has shown that hypoxia is a key microenvironment factor to promote the activation of PSCs (3). In pancreatic cancer, the accumulation of extracellular matrix leads to vascular collapse, impaired drug delivery and acquired chemoresistance, which may be induced by activated PSCs (4). Various factors secreted by stromal cells have recently been reported to promote gemcitabine (Gem) resistance (5). Gem is a nucleoside analogue, which has been the mainstay of pancreatic cancer chemotherapy for a long time. However, the clinical efficacy of Gem is limited due to its limited cellular uptake and impaired intracellular activation, resulting in low overall efficacy (6). Therefore, it is necessary to understand the biological characteristics of pancreatic cancer.

The hypoxic microenvironment, an important feature of solid tumors, promotes tumor cells to release exosomes (7). It is well known that exosomes secreted by multiple cells can mediate intracellular information transmission through delivering protein, messenger RNAs (mRNAs), microRNAs (miRNAs), and long noncoding RNAs (lncRNAs) between cells $(8,9)$. Interestingly, in order to adapt to the hypoxic microenvironment, many lncRNAs are highly expressed in tumor cells, encapsulated in exosomes, and transported to the corresponding receptor cells, thus conferring important roles in intercellular communication (7). For example, tumor cells can produce lncRNA UCA1-rich exosomes, which are transported to normoxic cells to promote tumor cell malignant phenotypes under hypoxia (10). Further, hypoxic exosomal lncRNA UCA1 has been found to promote drug resistance, angiogenesis, and tumor growth in pancreatic cancer $(7,11)$.

Moreover, lncRNA UCA1 is physically linked to the enhancer of zeste homolog 2 (EZH2), which inhibits p27Kip1 through histone methylation ( $\mathrm{H} 3 \mathrm{~K} 27 \mathrm{me} 3)$ in $\mathrm{p} 27 \mathrm{Kip} 1$ promoter, thus promoting tumorigenesis in nude mice in hepatocellular carcinoma (12). In addition, CTCF dependent recruitment of EZH2 to the SOCS3 gene promoter is likely to participate in the epigenetic silencing of SOCS3 and in regulating its gene expression in cancer development (13). SOCS3 overexpression has been observed to suppress malignant behaviors and Gem resistance of pancreatic cancer cells (14). Therefore, the present study set out to elucidate the roles of IncRNA UCA1 loaded in PSC-derived exosomes in Gem resistance of pancreatic cancer under hypoxia, and to identify the downstream mechanisms involving histone methylation in SOCS3 gene region and EZH2 recruitment.

\section{METHODS}

\section{Ethics Statement}

The study was conducted under the approval of the Ethics Committee of Shengjing Hospital of China Medical University. All participating patients signed informed consent documentation.
Animal experiments were approved by the Animal Ethics Committee of Shengjing Hospital of China Medical University. Extensive efforts were made to ensure minimal suffering of the included animals.

\section{Sample Collection}

From November 2018 to October 2019, the pancreatic cancer tissues and adjacent normal tissues $(5 \mathrm{~cm}$ from the edge of cancer tissue) of 35 patients with pancreatic cancer (aged 38-62 years old with a mean age of $52.8 \pm 5.9$ years; 23 males and 12 females) were collected in Shengjing Hospital of China Medical University. According to the staging classification proposed by Hermreck et al. (15), patients were clinically staged, with 10 cases of welldifferentiated, 14 cases of intermediate stage, and 11 cases of poorly differentiated. All pathological specimens were confirmed in the pathology department, and patients treated before surgery and patients without surgery were excluded. Tissue sections were frozen in liquid nitrogen and stored at $-80^{\circ} \mathrm{C}$.

\section{Bioinformatics Analysis}

Through the Gene Expression Omnibus (GEO) database (https:// www.ncbi.nlm.nih.gov/geo/), the pancreatic cancer related GSE32676 and GSE16515 datasets were downloaded with GPL570 and GPL10787 as the carrier platforms. GSE32676 dataset contains 7 normal samples and 25 pancreatic cancer samples, and GSE16515 dataset contains 16 normal samples and 36 pancreatic cancer samples. Differential expression analysis was performed using R language "limma" package (http://www. bioconductor.org/packages/release/bioc/html/limma.html), with $|\log \mathrm{FC}|>1$ and $\mathrm{p}$ value $<0.05$ set as the screening criteria to screen out the differentially expressed genes in pancreatic cancer and adjacent normal tissues.

\section{Cell Culture, Grouping, Transfection and Hypoxia Culture}

Human PSC cell line was obtained from the Mingzhou Biological Technology Co., Ltd. (Ningbo, China), and pancreatic cancer cell line PANC-1 and HEK-293T cells were purchased from Wuhan Procell Life Science \& Technology Co., Ltd. (Wuhan, China). All cells were cultured in Dulbecco's modified Eagle's medium (DMEM; Hyclone, Beijing, China) containing 10\% fetal bovine serum (FBS; Gibco, Invitrogen), $100 \mathrm{mg} / \mathrm{mL}$ penicillin and 100 $\mathrm{mg} / \mathrm{mL}$ streptomycin at $37^{\circ} \mathrm{C}$ in $5 \% \mathrm{CO}_{2}$ and $95 \%$ saturated humidity. When the cell confluence was about $80 \%$, the cells were passaged. HPSCs were cultured under the same conditions as normal pancreatic epithelial cells and pancreatic cancer cell lines. Hypoxia was induced by $12-\mathrm{h}$ incubation of PSCs in a nitrogen-filled incubator $\left(94 \% \mathrm{~N}_{2}, 5 \% \mathrm{CO}_{2}, 1 \% \mathrm{O}_{2}\right.$ ) (Thermo Fisher Scientific Inc., Waltham, MA), and obtained hypoxic PSCs were hereinafter referred as HPSCs. Three biological replicates were set for each group.

Before transfection, the cells in logarithmic phase were seeded into 6-well plates at a density of $1 \times 10^{5}$ cells/well. The cells were transfected with lentiviral vectors (Invitrogen) according to the instructions of Lipofectamine 2000 transfection reagent (11668019, Invitrogen) (Supplementary Table 1). 


\section{Extraction and Identification of Exosomes}

Exosomes in human PSCs and HPSCs (PSCs-EXO, HPSC-EXO) were isolated and extracted by total exosome kit (Thermo Fisher Scientific). The specific operation was as follows: the culture supernatant was collected, centrifuged at $4^{\circ} \mathrm{C}, 2000 \mathrm{~g}$ for $30 \mathrm{~min}$, and the cells and debris were removed. The supernatant (4000 g) was centrifuged at room temperature for $15 \mathrm{~min}$ to enrich the exosomes, and then mixed with 0.5 -fold volume of total exosomes separation reagent. The mixture was incubated overnight at $4^{\circ} \mathrm{C}$ and then centrifuged at $4^{\circ} \mathrm{C}$ for $60 \mathrm{~min}$ the next day. The precipitated exosomes were isolated and resuspended with PBS or stored at $-80^{\circ} \mathrm{C}$.

Identification: BCA protein detection kit (product No. 23228; Thermo Fisher Scientific) was used to determine the protein concentration in PSCs-EXO and HPSC-EXO. In addition, the protein levels of CD9 (Abcam, ab92726, 1:2000) and CD63 (Abcam, ab59479, 1:1000) were detected by Western blot. The exosome morphology and size were observed by a transmission electron microscope (TEM; Morgagni 268D, Philips, Holland). The size and size distribution of the PSCs-EXO and HPSC-EXO were detected by Malvern NanoSight NS300.

TEM observation: $20 \mu \mathrm{L}$ fresh samples of PSCs-EXO and HPSC-EXO were loaded into the carbon coated copper electron microscope for $2 \mathrm{~min}$, and then negatively stained with phosphotungstic acid solution (12501-23-4, Sigma-Aldrich) for 5 min. The EVs were then washed three times with PBS to remove the excess phosphotungstic acid solution, and then kept semi dry with filter paper. The image was observed at $80 \mathrm{KV}$ in Hitachi H-7650 TEM (Hitachi High-Tech, Tokyo, Japan).

Nanosight nanoparticle size analysis: $20 \mu \mathrm{g}$ PSCs-EXO and HPSC-EXO was dissolved in $1 \mathrm{~mL}$ PBS for $1 \mathrm{~min}$, and the uniform distribution of PSCs-EXO and HPSC-EXO was maintained. Then, the particle size distribution of PSCs-EXO and HPSC-EXO was directly observed and measured by nanosight nanoparticle tracking analyzer (NTA, Malvern Instruments, Malvern, UK).

\section{MTT Assay}

MTT method (Dojindo, JPN) was used to analyze cell proliferation. PANC-1 cells in logarithmic growth phase were seeded into 96-well culture plate at a density of $1 \times 10^{3}$ cells/well. The cells were then transfected and placed in a cell incubator with 3 repeated wells in each group. After the cells were cultured for $96 \mathrm{~h}$, each well was added with $100 \mu \mathrm{L}$ of $0.5 \mathrm{mg} / \mathrm{mL}$ MTT buffer and cultured in an incubator for $2 \mathrm{~h}$. A plate reader (Bio-Rad Laboratories, Hercules, CA) was then used to measure the OD value of each well at $490 \mathrm{~nm}$.

For the gemcitabine response of PANC-1 cells, PANC-1 cells were pretreated according to the instructions and seeded in a 96well plate at a density of 3000 cells per well, with 3 replicate wells in each group. After $12 \mathrm{~h}$, the cells were incubated with $10 \mathrm{nM}$ Gem (G6423, Sigma-Aldrich) for 1, 2, 3, 4, and 5 days. The cells were then incubated with $100 \mu \mathrm{L}$ of $0.5 \mathrm{mg} / \mathrm{mL}$ MTT buffer for $2 \mathrm{~h}$. The OD value of each well at $490 \mathrm{~nm}$ was measured using a multi-well plate reader (Bio-Rad).

\section{Flow Cytometry}

After $48 \mathrm{~h}$ of transfection, the cells were digested with $0.25 \%$ trypsin (without EDTA) and centrifuged. According to the instructions of Annexin-V-FITC cell apoptosis detection kit
(556547, Shanghai shuojia Biotechnology Co., Ltd., Shanghai, China), the cells were resuspended in $500 \mu \mathrm{L}$ Binding Buffer and incubated with $5 \mu \mathrm{L}$ Annexin V-FITC at room temperature for $15 \mathrm{~min}$, followed by incubation with $10 \mu \mathrm{L}$ PI on ice for $5 \mathrm{~min}$ in the dark. Next, $1 \times 10^{6}$ cells were resuspended in $100 \mu \mathrm{L}$ of dye solution. After incubation at room temperature for $15 \mathrm{~min}, 1 \mathrm{~mL}$ of HEPES buffer solution was added. The fluorescence of FITC and PI was detected at $515 \mathrm{~nm}$ and $620 \mathrm{~nm}$ respectively.

\section{qRT-PCR}

TRIzol reagent (Thermo Fisher Scientific) was used to extract total RNA from tissues and cells. Then $1 \mu \mathrm{g}$ total RNA was reversely transcribed into cDNA using reverse transcription kit PrimeScript RT reagent Kit with gDNA Eraser (TaKaRa, Japan). qRT-PCR was performed using SYBR Premix Ex Taq II (TaKaRa) in an ABI 7500 qPCR instrument (Thermo Fisher Scientific) with glyceraldehyde-3-phosphate dehydrogenase (GAPDH) serving as internal reference. The designed primers are shown in Supplementary Table 2. The relative transcription expression of target genes was calculated via relative quantitative method ( $2^{-\Delta \Delta C T}$ method).

\section{Western Blot}

The total protein was extracted by radioimmunoprecipitation assay (RIPA) lysate (Beijing Solarbio Science \& Technology Co., Ltd., Beijing, China), and the protein concentration was determined by BCA kit (GBCBIO Technologies, Guangdong, China). The proteins were separated by polyacrylamide gel electrophoresis and transferred to polyvinylidene fluoride membranes (Millipore Corp., Billerica, MA), and 5\% BSA was used to seal the proteins at room temperature for $1 \mathrm{~h}$. Diluted primary rabbit antibodies (Supplementary Table 3 ) were used to incubate the proteins overnight at $4^{\circ} \mathrm{C}$ with $\mathrm{GAPDH}$ used as internal reference. The next day, secondary antibody goat antirabbit IgG antibody was added to the membranes and incubated for $2 \mathrm{~h}$. The membranes were then reacted with enhanced chemiluminescence solution, developed in Image Quant LAS 4000C gel imager (GE Healthcare, Little Chalfont, UK), and analyzed using Quantity One v4.6.2 software.

\section{Fluorescent Labeling and Transfer of Exosomes}

Cells at the logarithmic growth phase were collected for this experiment. The isolated exosomes were incubated with lipophilic dye $\mathrm{DiO}$ (Thermo Fisher Scientific) at a working concentration of $10 \mu \mathrm{M}$ at $37^{\circ} \mathrm{C}$ for $20 \mathrm{~min}$, and centrifuged at $12000 \times \mathrm{g}$ and $4^{\circ} \mathrm{C}$ for $70 \mathrm{~min}$. The PANC- 1 cells $\left(3 \times 10^{4}\right.$ cells per well) were incubated with DiO-labeled exosomes $(25 \mu \mathrm{g} / \mathrm{mL})$ for $24 \mathrm{~h}$.

In order to confirm the transfer of lncRNA UCA1, HPSCs were transfected with Cy3-labeled IncRNA UCA1. HPSCs and PANC-1 cells expressing Cy3-lncRNA UCA1 were co-cultured for $48 \mathrm{~h}$ in a 24-well Transwell chamber. Next, PANC-1 cells were prepared as described above for immunofluorescence. In addition, the cytoskeleton of PANC-1 cells was stained with TRITC-Phalloidin (100 nM, 40734ES75, Yeasen Company, Shanghai, China) or FITC Phalloidin (100 nM, 40734ES80, 
Yeasen). Finally, a confocal microscope was used to determine the internalization of exosomes or exosomal lncRNA UCA1.

\section{Transwell Assay for Cell Invasion and Migration}

Transwell chamber (pore size of $8 \mathrm{~mm}$; Corning Incorporated, Corning, NY) in 24-well plates was used for this experiment. For the invasion experiment, $100 \mu \mathrm{L}$ of Matrigel (BD Biosciences) was placed in each chamber before the experiment and solidified at $37^{\circ} \mathrm{C}$ for $2 \mathrm{~h}$. The cells were digested, washed twice with PBS, and resuspended in serum-free DMEM/F12 medium to a density of $3 \times 10^{5}$ cells $/ \mathrm{mL}$. Each group had 3 chambers, $200 \mu \mathrm{L}$ cell suspension for each. Thereafter, $700 \mu \mathrm{L} 10 \%$ DMEM was added to the lower chamber and incubated in a $37^{\circ} \mathrm{C}$ incubator with $5 \%$ $\mathrm{CO}_{2}$. After $48 \mathrm{~h}$, the cells were fixed with $4 \%$ paraformaldehyde for $30 \mathrm{~min}$, and stained with $0.05 \%$ crystal violet (G1062, Solarbio) for 5 min. The invaded cells were counted and photographed under an Olympus IX 71 microscope (Olympus, Tokyo, Japan).

\section{TUNEL Staining}

In situ apoptosis detection kit (KeyGen BioTech Ltd, Jiangsu, China) was used to detect genomic DNA breakage during cell apoptosis. The sample was incubated with $100 \mu \mathrm{L}$ of proteinase $\mathrm{K}$ solution at $37^{\circ} \mathrm{C}$ for $30 \mathrm{~min}$. Next, $50 \mu \mathrm{L}$ of TdT enzyme solution was added to the cell sample and incubated at $37^{\circ} \mathrm{C}$ in the dark for $60 \mathrm{~min}$. Following this, the cells were incubated with $5 \mu \mathrm{L}$ of streptavidin fluorescein solution and $45 \mu \mathrm{L}$ of labeling buffer in the dark at $37^{\circ} \mathrm{C}$ for $30 \mathrm{~min}$ and stained with DAPI (SigmaAldrich) before observation under a fluorescence microscope.

\section{Chromatin Immunoprecipitation}

Cells $\left(5 \times 10^{6}\right)$ were fixed in $1 \%$ formaldehyde for $30 \mathrm{~min}$ at room temperature and subjected to ultrasonic treatment to produce 150-900 bp chromatin fragments. The chromatin was then immunoprecipitated with antibodies to EZH2, H3K27me3 or normal rabbit IgG (serving as NC). qRT-PCR was used to quantify the purified chromatin. The signals obtained from the ChIP assay were divided by the signals obtained from an Input sample. In this assay, $1 \%$ of starting chromatin served as the Input, and a dilution factor of 100 or 6.644 cycles $(\log 2$ of 100) was subtracted from the Ct value of the diluted Input. The primer is shown in Supplementary Table 4.

\section{RNA-Binding Protein Immunoprecipitation Assay}

TRIzol reagent (Thermo Fisher Scientific) was used to extract total RNA from PSC cells. mRNA in the total RNA was isolated and purified by PolyATtract ${ }^{\circledR}$ mRNA Isolation Systems (AZ5300, A\&D Technology Corporation, Beijing, China). AntiEZH2 antibody (36-6300, Thermo Fisher Scientific) or anti-IgG antibody (ab124055, Abcam) was added into IP buffer (20-mM Tris $\mathrm{pH} 7.5,140-\mathrm{mM} \mathrm{NaCl}, 1 \% \mathrm{NP}-40,2-\mathrm{mM}$ EDTA) to incubate with protein $\mathrm{A} / \mathrm{g}$ magnetic beads for $1 \mathrm{~h}$ for binding. The purified mRNA and magnetic bead-antibody complex were added into IP buffer supplemented with ribonuclease inhibitor and protease inhibitor, and were kept overnight at $4^{\circ} \mathrm{C}$. The RNA was eluted with eluent buffer. After extraction and purification by phenol chloroform, IncRNA UCA1 was analyzed by qRT-PCR.

\section{Establishment of Gem-Resistant Cell Line}

A Gem-resistant cell line was induced in MIA PaCa-2 pancreatic cancer cell line by increasing the concentration of Gem $(0.25 \mu \mathrm{M}$, $1 \mu \mathrm{M}$ or $3 \mu \mathrm{M})$ in the medium. It took about a week for cells to acquire resistance and resume cell proliferation with each increase of Gem concentration. The whole process took several months to reach the most resistant cell line GemMIA-R3 with the highest Gem concentration $(3.0 \mu \mathrm{m})$. At each point, the cells were amplified, cryopreserved, and maintained at a concentration in a medium containing $3.0 \mu \mathrm{M}$. Prior to the use of specific anti-3.0 $\mu \mathrm{M}$ cell line in any experiment, Gem selection pressure was relieved for 7 days to avoid Gem interference. The concentration response curve and cell growth inhibition data are shown in Supplementary Figure 1.

\section{Pancreatic Cancer Xenografting Mice Model}

Four-to-six-week-old NOD/SCID mice were raised under specific pathogen-free conditions at $26-28^{\circ} \mathrm{C}$ and $50-65 \%$ humidity. The grouping of mice was consistent with the cell grouping (Supplementary Table 1). Cancer cells in the pancreas were counted and resuspended in PBS at a final concentration of $2 \times$ $10^{7}$ cells $/ \mathrm{mL}$. In addition, trypan blue exclusion method determined that $95 \%$ of the cells were viable before injection. The cells were then subcutaneously injected into the NOD/SCID mice. At the same time, $20 \mu \mathrm{g}$ exosomes were injected into the mice via tail vein every three days. Two weeks after implantation, the mice were treated with $80 \mathrm{mg} / \mathrm{kg} \mathrm{Gem}$ (G6423; Sigma-Aldrich) via intraperitoneal injection, three times a week for five weeks. The tumor volume was measured with Vernier calipers every week and calculated using the following formula: tumor volume $=1 / 2 \times a \times$ $b^{2}$ ( $a$ is long diameter and $b$ is short diameter). After 30 days, the mice were euthanized, and the tumor tissues were collected and weighed. Meanwhile, paraffin embedded tissues were prepared for subsequent experimental analysis.

\section{Immunohistochemistry}

Tumor tissues were fixed in 10\% neutral formalin, embedded in paraffin and cut into $5-\mu \mathrm{m}$-thick sections. Immunohistochemistry was performed on a Ventana Discovery Ultra automatic dyeing machine (Ventana Medical Systems, Roche Diagnostics, Indianapolis). The cover glass was loaded on the machine whereupon the sections were dewaxed and rehydrated, followed by endogenous peroxidase activity elimination and antigen retrieval. The sections were incubated with anti-Ki67 antibody (ab16667, Abcam), developed with DISCOVERY ChromoMap $\mathrm{DAB}$ Kit and counterstained with hematoxylin (Ventana). The images were collected with a DMi8 Leica microscope and analyzed with Image-Pro Premier software.

\section{Statistical Analysis}

Statistical analysis was conducted using the SPSS 21.0 software (IBM, Armonk, New York). Measurement data were expressed as mean \pm standard deviation. The data between two groups were 
compared using the independent sample $t$ test. Paired $t$ test was used for comparison between cancer tissues and adjacent normal tissues. The data comparison among multiple groups was performed using one-way analysis of variance (ANOVA), followed by the Tukey's post hoc test. The data at different time points were compared by repeated measures ANOVA, followed by Bonferroni's post hoc test. Pearson's correlation coefficient was used for correlation analysis. A value of $p<$ 0.05 was indicative of a statistically significant difference.

\section{RESULTS}

\section{Hypoxia Induces Activation of PSCs and Promotes the Release of Exosomes}

Evidence exists reporting that hypoxia can induce the activation of PSCs (3), but the mechanism is unclear. In order to understand the effect of hypoxia on the activation of PSCs, we assayed the activities of PSCs cultured in normal environment and HPSCs cultured in hypoxia. The MTT results (Figure 1A) showed that compared with PSCs, hypoxia induced higher viability in HPSCs. Consistently, the expression of hypoxia inducible factor- $1 \alpha$ (HIF-1 $\alpha$ ), as measured by qRT-PCR and Western blot, was up-regulated in HPSCs relative to PSCs (Figures 1B, C), which was consistent with the published literature $(3,16)$. The isolated PSC-EXO and HPSC-EXO were observed by a TEM, and the typical double-layer membrane and cup-shaped structure of exosomes could be observed (Figure 1D). Meanwhile, NTA (Figure 1E) showed irregular Brownian motion and an average diameter of $96.1 \mathrm{~nm}$ and 106.5 $\mathrm{nm}$ for the size distribution of PSC-EXO and HPSC-EXO. In addition, Western blot data (Figure 1F) showed that exosome marker proteins (CD9 and CD63) were significantly expressed in exosomes.
A

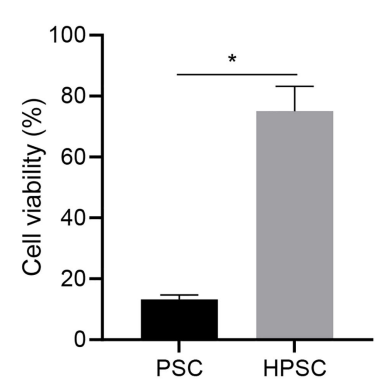

D
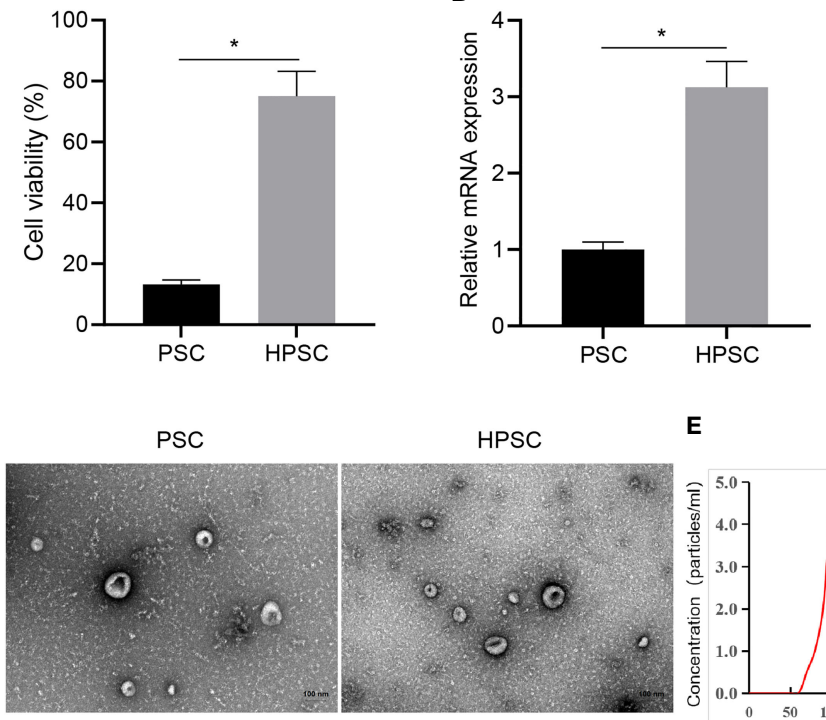

E

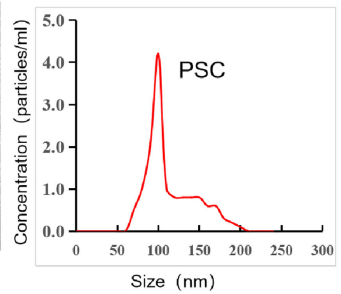

G

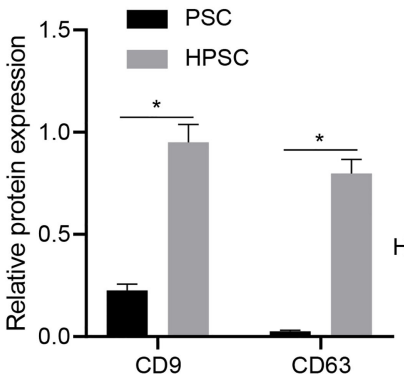

TRITC Phalloidin

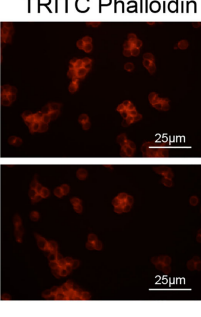

DiO-Exos

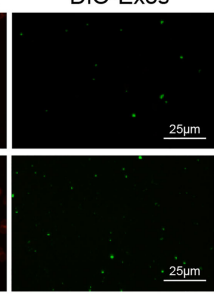

C
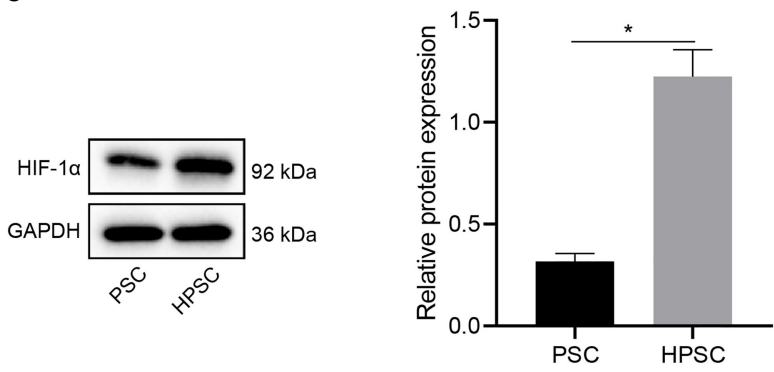

$\mathbf{F}$
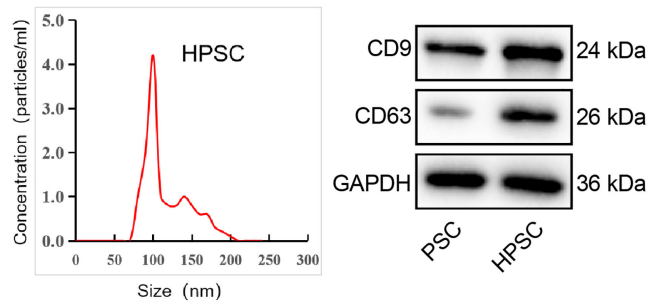

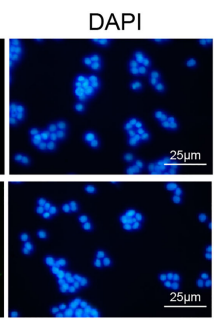

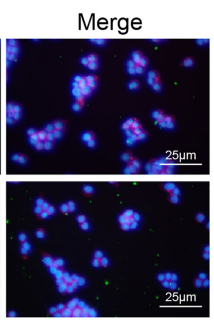

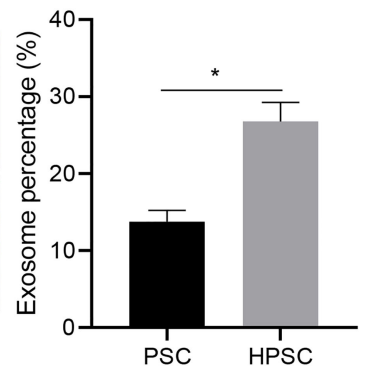

FIGURE 1 | Hypoxia induces PSC activation and exosome release. (A), Viability of PSCs and HPSCs was detected by MTT assay. (B), Expression of HIF-1 in PSCs and HPSCs was detected by GRT-PCR. (C), Expression of HIF-1 in PSCs and HPSCs was detected by Western blot. (D), Morphology of exosomes isolated from PSCs and HPSCs (PSC-EXO and HPSC-EXO) observed under a TEM. (E), NTA of the size distribution of PSC-EXO and HPSC-EXO. (F), Expression of exosome marker protein in PSC-EXO and HPSC-EXO detected by Western blot. (G), Immunofluorescence analysis of the uptake of DiO-labbeled PSC-EXO and HPSC-EXO by PANC-1 cells. ${ }^{*} p<0.05$. Measurement data were expressed as mean \pm standard deviation. Independent sample $t$ test was applied for the comparison of data of two groups. The cell experiment was repeated three times. 
To determine whether the PSC-EXO and HPSC-EXO were absorbed by pancreatic cancer cells, exosomes were labeled with $\mathrm{DiO}$ and cytoskeleton with TRITC Phalloidin, followed by coculture with PANC-1 cells for $24 \mathrm{~h}$. Confocal microscopic observation results showed that DiO-labeled exosomes were internalized by PANC-1 cells (Figure 1G). The above results demonstrate the successful isolation of the exosomes and that hypoxia could induce PSC activation and exosome release.

\section{HPSC-EXO Promotes Gem Resistance and Tumorigenesis of Pancreatic Cancer Cells}

PANC-1 cells were treated with HPSC-EXO or PSC-EXO to determine the possible effect of exosomes on the biological functions of PANC-1 cells. MTT assay results (Figure 2A) found that the cell viability in the presence of HPSC-EXO was the highest. Meanwhile, the results of flow cytometry (Figure 2B) and TUNEL staining (Figure 2C) showed that PSC-EXO treatment had no effect on cell apoptosis but HPSC-EXO treatment inhibited PANC-1 cell apoptosis. In addition, Western blot results (Figure 2D) also showed that the expression of cleaved caspase 3 was diminished only in the presence of HPSC-EXO, as compared with PBS treatment. The results of Transwell assay revealed that only HPSC-EXO treatment enhanced the migration and invasion of PANC-1 cells (Figures 2E, F).

Next, we tested the effect of HPSC-EXO on the gemcitabine resistance of PANC-1 cells. PANC-1 cells were incubated with Gem $(10 \mu \mathrm{M})$, the proliferation of which was determined by MTT assay. The results suggested that HPSC-EXO promoted the resistance of PANC-1 cells to Gem, while PSC-EXO showed no influence on the cell resistance (Figure 2G).

Next, we shifted to further verify the effect of HPSC-EXO on pancreatic cancer cells in vivo. As shown in Figures $2 \mathbf{H}-\mathbf{J}$, HPSC-EXO treatment led to higher tumor volume and weight of mice and positive rate of the proliferation marker Ki67 in the tumor tissues than PBS treatment while PSC-EXO treatment showed no influence. In addition, HPSC-EXO treatment also significantly alleviated the inhibition of Gem on the tumor growth and Ki67 expression. These results suggest that HPSCEXO can accelerate the tumorigenesis of pancreatic cancer cells and Gem resistance.

\section{LncRNA UCA1-Loaded HPSC-EXO Promote the Malignant Phenotypes and Gem Resistance of Pancreatic Cancer Cells}

To further explore the specific mechanism of HPSC-EXO in promoting Gem resistance and tumorigenesis in pancreatic cancer, we performed differential expression analysis on the GSE32676 and GSE16515 datasets. The results showed that lncRNA UCA1 was highly expressed in pancreatic cancer samples (Figures 3A, B). Therefore, we speculated that HPSCEXO may affect the biological functions of PANC-1 cells through IncRNA UCA1. The results of qRT-PCR showed that the expression of lncRNA UCA1 was significantly higher in HPSCs and HPSC-EXO than that in PSCs and PSC-EXO, respectively (Figure 3C). At the same time, compared with the
PBS treatment, HPSC-EXO treatment increased the expression of lncRNA UCA1 in PANC-1 cells but there was no significant alteration in PANC-1 cells treated with PSC-EXO (Figure 3D). In addition, HPSCs were transfected with Cy3-labeled lncRNA UCA1 and then co-cultured with PANC-1 cells for $48 \mathrm{~h}$. The observation results using a confocal microscope suggested that fluorescently labeled lncRNA UCA1 could be observed in PANC-1 cells (Figure 3E). These results indicate that HPSCEXO transferred lncRNA UCA1 to PANC-1 cells.

Next, we set up three IncRNA UCA1 shRNA sequences. qRTPCR results showed that sh-lncRNA UCA1-3 had the most significant effect on inhibiting the expression of lncRNA UCA1 (Figure 3F). Therefore, sh-lncRNA UCA1-3 was selected for subsequent experiments. qRT-PCR results showed an increase in the expression of lncRNA UCA1 in PANC-1 cells with lncRNA UCA1 silencing and a more pronounced increase was noted in sh-lncRNA UCA1-treated PANC-1 cells co-cultured with HPSC-EXO (Figure 3G). MTT assay results revealed reduced viability of PANC-1 cells after lncRNA UCA1 knockdown, which was restored by further co-culture with HPSC-EXO (Figure 3H). Moreover, the results of flow cytometry (Figure 3I) and TUNEL staining (Figure 3J) showed that shlncRNA UCA1 treatment alone promoted the apoptosis of PANC-1 cells, which were reversed by additional co-culture with HPSC-EXO. Western blot data also showed that the expression of cleaved caspase 3 was increased following shlncRNA UCA1 treatment in PANC-1 cells, while it was reduced after further co-culture with HPSC-EXO (Figure 3K). Furthermore, Transwell assay results indicated a decline in the migration and invasion of cells following lncRNA UCA1 knockdown while treatment with HPSC-EXO + sh-lncRNA UCA1 abolished this effect (Figures 3L, M). As shown in Figure 3N, PANC-1 cells with lncRNA UCA1 knockdown were more sensitive to Gem while further co-culture with HPSC-EXO reversed this sensitivity to a certain extent.

These results reveal that lncRNA UCA1 can be transferred to pancreatic cancer cells by HPSC-EXO, thereby promoting the malignant phenotypes of pancreatic cancer cells and their resistance to Gem.

\section{LncRNA UCA1 Recruits EZH2 to the Promoter of SOCS3 and Downregulates SOCS3 Expression}

We then moved to illustrate the downstream mechanism of the effect of IncRNA UCA1 in pancreatic cancer. Differential expression analysis on the GSE32676 and GSE16515 datasets showed that SOCS3 was poorly expressed in pancreatic cancer samples (Figures 3A, B). The results of qRT-PCR showed higher expression of IncRNA UCA1 and EZH2 and lower SOCS3 expression in pancreatic cancer tissues than that in adjacent normal tissues (Figures 4A-C). In addition, Pearson's correlation analysis showed that SOCS3 expression was negatively correlated with the expression ofEZH2 $(r=-0.75, p<0.01)$ and $\operatorname{lncRNA}$ UCA1 $(\mathrm{r}=-0.73, p<0.01)$ in pancreatic cancer tissues (Figure 4D).

Next, we sought to explore the regulatory relationship between EZH2 and SOCS3. In PANC-1 cells, although the 
A

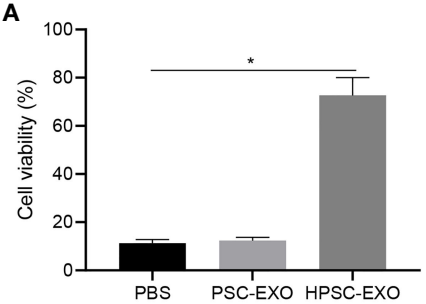

D

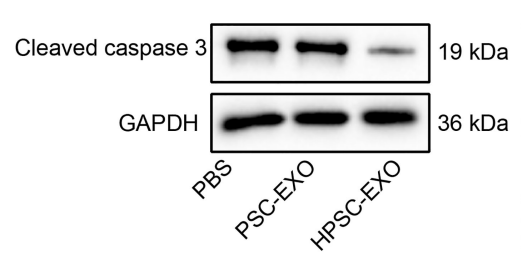

$\mathbf{F}$

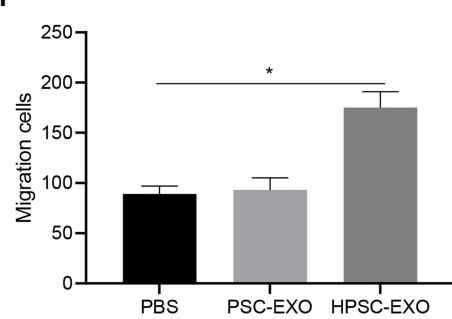

B

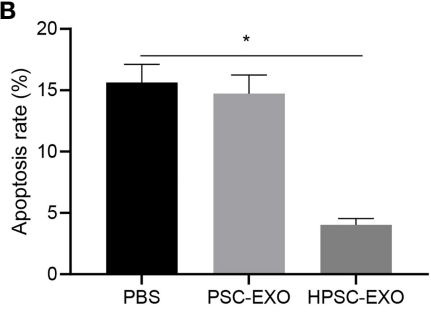

E

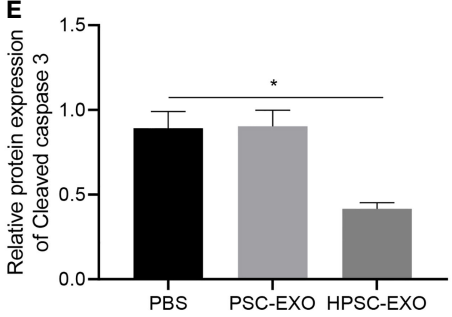

G $\rightarrow$ PBS

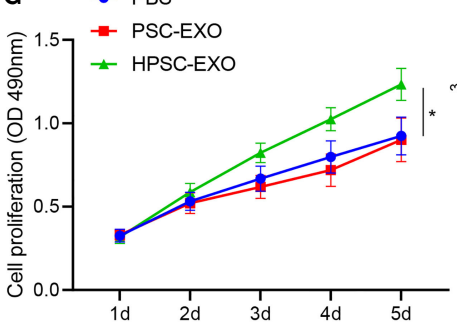

PBS
C
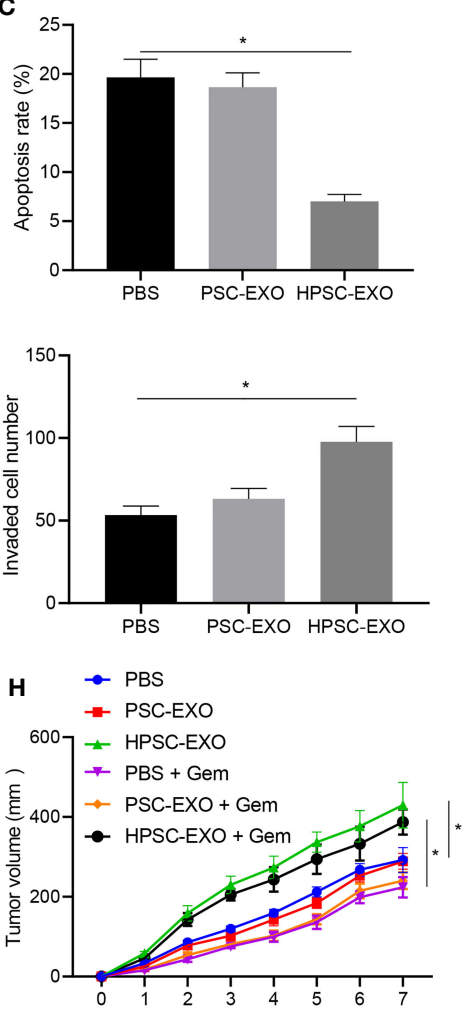

I

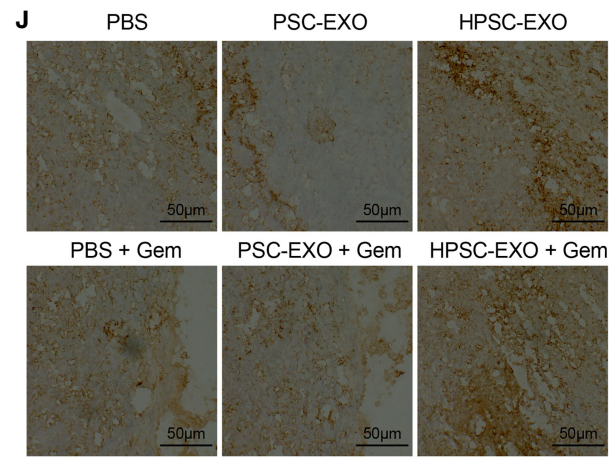

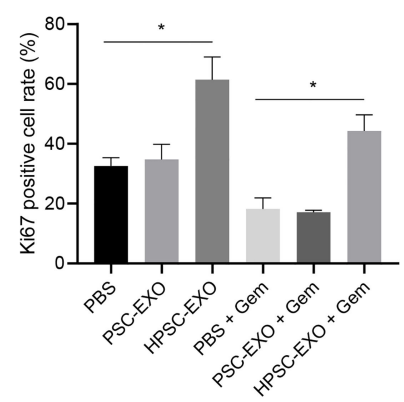

FIGURE 2 | HPSC-EXO facilitates Gem resistance and tumorigenesis of pancreatic cancer cells. (A), Viability of PANC-1 cells treated with PSC-EXO and HPSC-EXO was detected by MTT assay. (B), Flow cytometric analysis of apoptosis of PANC-1 cells treated with PSC-EXO and HPSC-EXO. (C), Apoptosis of PANC-1 cells treated with PSC-EXO and HPSC-EXO was detected by TUNEL staining. (D), Cleaved caspase 3 protein expression in PANC-1 cells treated with PSC-EXO and HPSC-EXO was detected by Western blot. (E), Migration of PANC-1 cells treated with PSC-EXO and HPSC-EXO was detected by Transwell assay. (F), Invasion of PANC-1 cells treated with PSC-EXO and HPSC-EXO was detected by Transwell assay. (G), Gem sensitivity of PANC-1 cells treated with PSC-EXO and HPSC-EXO was detected by MTT assay. (H), Tumor volume of mice treated with PSC-EXO and HPSC-EXO after injection of Gem. (I), Tumor weight of mice treated with PSCEXO and HPSC-EXO after injection of Gem. (J), The positive expression rate of Ki67 in tumor tissues of mice treated with PSC-EXO and HPSC-EXO after injection of Gem was detected by immunohistochemistry. ${ }^{*} p<0.05$. Measurement data were expressed as mean \pm standard deviation. The data comparison among multiple groups was performed using one-way ANOVA, followed by the Tukey's post hoc test. The data at different time points were compared by repeated measures ANOVA, followed by Bonferroni's post hoc test. The cell experiment was repeated three times. $\mathrm{n}=8$ for mice following each treatment.

total expression of EZH2 did not change, the level of $\mathrm{H} 3 \mathrm{~K} 27 \mathrm{me} 3$ in response to sh-lncRNA UCA1 was reduced to the level equivalent to that of GSK-126 (EZH2 inhibitor) (Figure 4E). Consistently, ChIP results showed that the binding of EZH2 and $\mathrm{H} 3 \mathrm{~K} 27 \mathrm{me} 3$ in the SOCS3 promoter was reduced in PANC-1 cells in response to sh-lncRNA UCA1 or GSK-126 (Figures 4F, G). In addition, RIP assay results showed that lncRNA UCA1 bound to EZH2 in PANC-1 cells (Figure $\mathbf{4 H}$ ). Western blot results also showed that knockdown of lncRNA UCA1 in PANC-1 cells elevated the expression of SOCS3 (Figure 4I). These data suggest that lncRNA UCA1 is involved in EZH2 mediated epigenetic inhibition of SOCS3 in PANC-1 cells by binding to EZH2. 
A

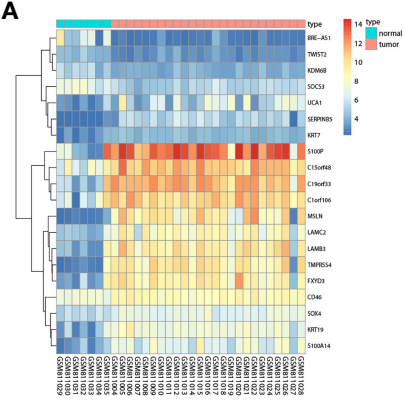

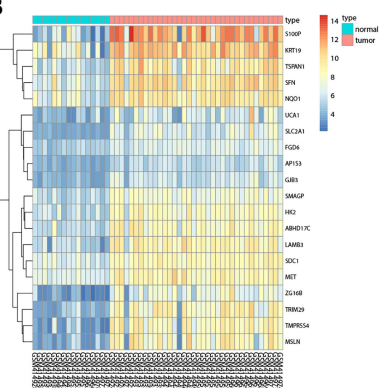

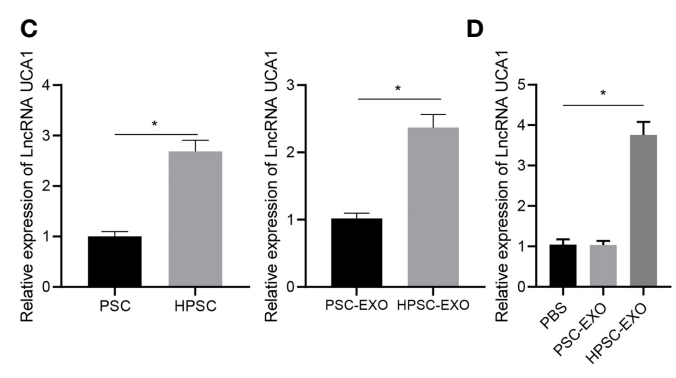

C

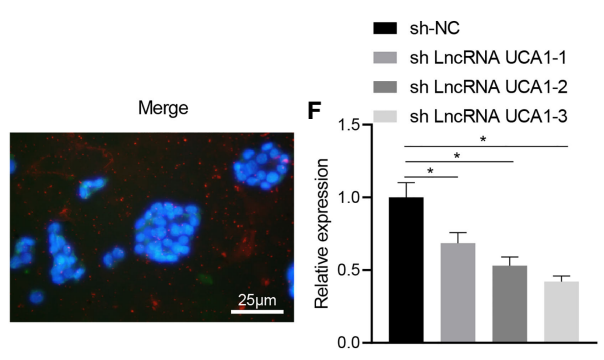

E DAPI
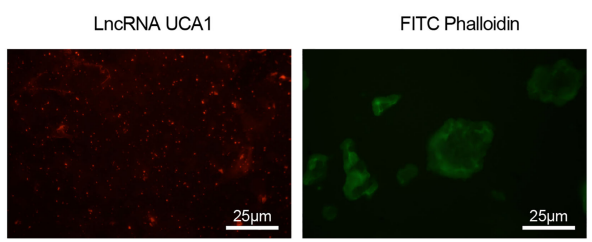

\section{I sh-NC}

- PSC EXO-sh-NC

- sh LnCRNA UCA1

$\mathbf{J}$ sh-NC

- PSC EXO-sh-NC

- sh LnCRNA UCA1

- PSC EXO-sh-NC

sh LnCRNA UCA1

* HPSC EXO-sh LncRNa UCA1

- HPSC EXO-sh LncRNA UCA1

- PSC EXO-sh-NC

- sh LnCRNA UCA1

- HPSC EXO-sh LnCRNA UCA1
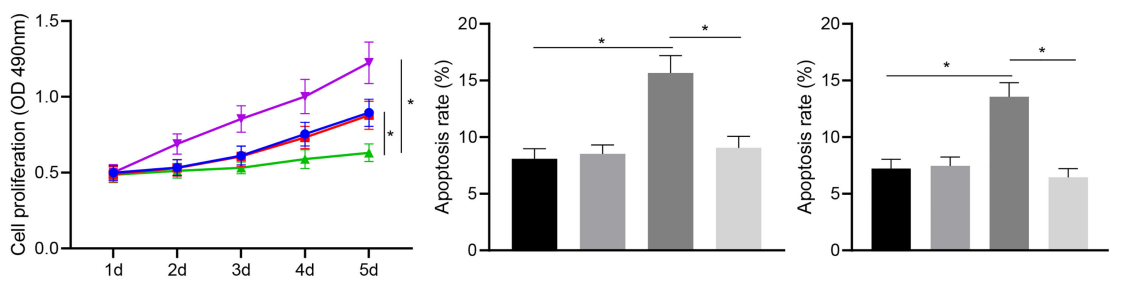

K
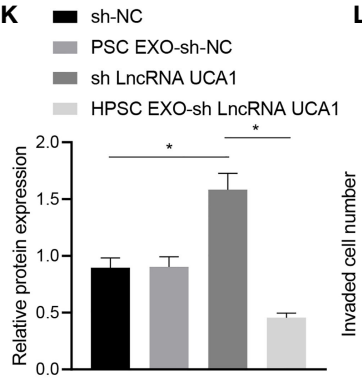

L sh-NC

- PSC EXO-sh-NC

- sh LncRNA UCA1

HPSC EXO-sh LncRNA UCA1

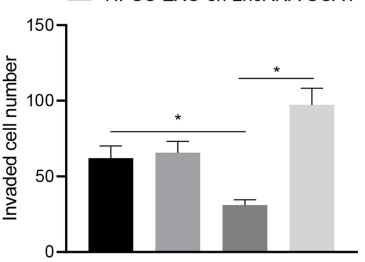

M
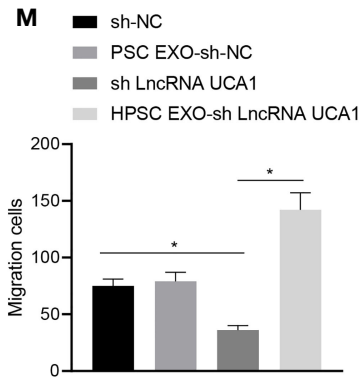

N $\rightarrow$ sh-NC

- PSC EXO-sh-NC

\# sh LnCRNA UCA1

* HPSC EXO-sh LncRNA UCA1

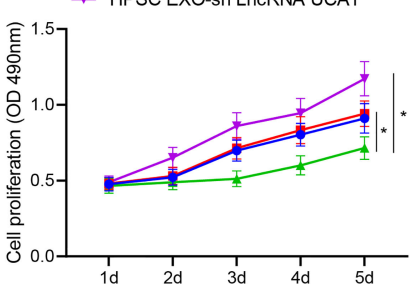

FIGURE 3 | HPSC-EXO transfer IncRNA UCA1 to pancreatic cancer cells, where IncRNA UCA1 stimulates the malignant phenotypes of pancreatic cancer cells and their resistance to Gem. (A), A heatmap of the expression of the first 10 differentially expressed genes in pancreatic cancer samples in the GSE32676 dataset. (B), A heatmap of the expression of the first 10 differentially expressed genes in pancreatic cancer samples in the GSE16515 dataset. (C), Expression of IncRNA UCA1 in PSCs and HPSCs as well as their derived exosomes was detected by qRT-PCR. (D), Expression of IncRNA UCA1 in PANC-1 cells treated with PSC-EXO and HPSC-EXO was detected by qRT-PCR. (E), Co-localization of IncRNA UCA1 and exosomes in PANC-1 cells was observed by a fluorescence microscope.

(F), Transfection efficiency of three IncRNA UCA1 shRNA sequences in PANC-1 cells was detected by qRT-PCR. (G), Expression of IncRNA UCA1 in the PANC-1 cells treated with sh-IncRNA UCA1 or combined with HPSC-EXO was detected by qRT-PCR. (H), Viability of PANC-1 cells treated with sh-IncRNA UCA1 or combined with HPSC-EXO was detected by MTT assay. (I), Apoptosis of PANC-1 cells treated with sh-IncRNA UCA1 or combined with HPSC-EXO was detected by flow cytometry. (J), Apoptosis of PANC-1 cells treated with sh-IncRNA UCA1 or combined with HPSC-EXO was detected by TUNEL staining. (K), Cleaved caspase 3 protein expression in the PANC-1 cells treated with sh-IncRNA UCA1 or combined with HPSC-EXO was detected by Western blot. (L), Migration of PANC-1 cells treated with sh-IncRNA UCA1 or combined with HPSC-EXO was detected by Transwell assay. (M), Invasion of PANC-1 cells treated with sh-IncRNA UCA1 or combined with HPSC-EXO was detected by Transwell assay. (N), Gem sensitivity of PANC-1 cells treated with sh-IncRNA UCA1 or combined with HPSC-EXO was detected by MTT assay. ${ }^{*} p<0.05$. Measurement data were expressed as mean \pm standard deviation. The data between two groups were compared using the independent sample $t$ test. The data comparison among multiple groups was performed using one-way ANOVA, followed by the Tukey's post hoc test. The data at different time points were compared by repeated measures ANOVA, followed by Bonferroni's post hoc test. The cell experiment was repeated three times. 


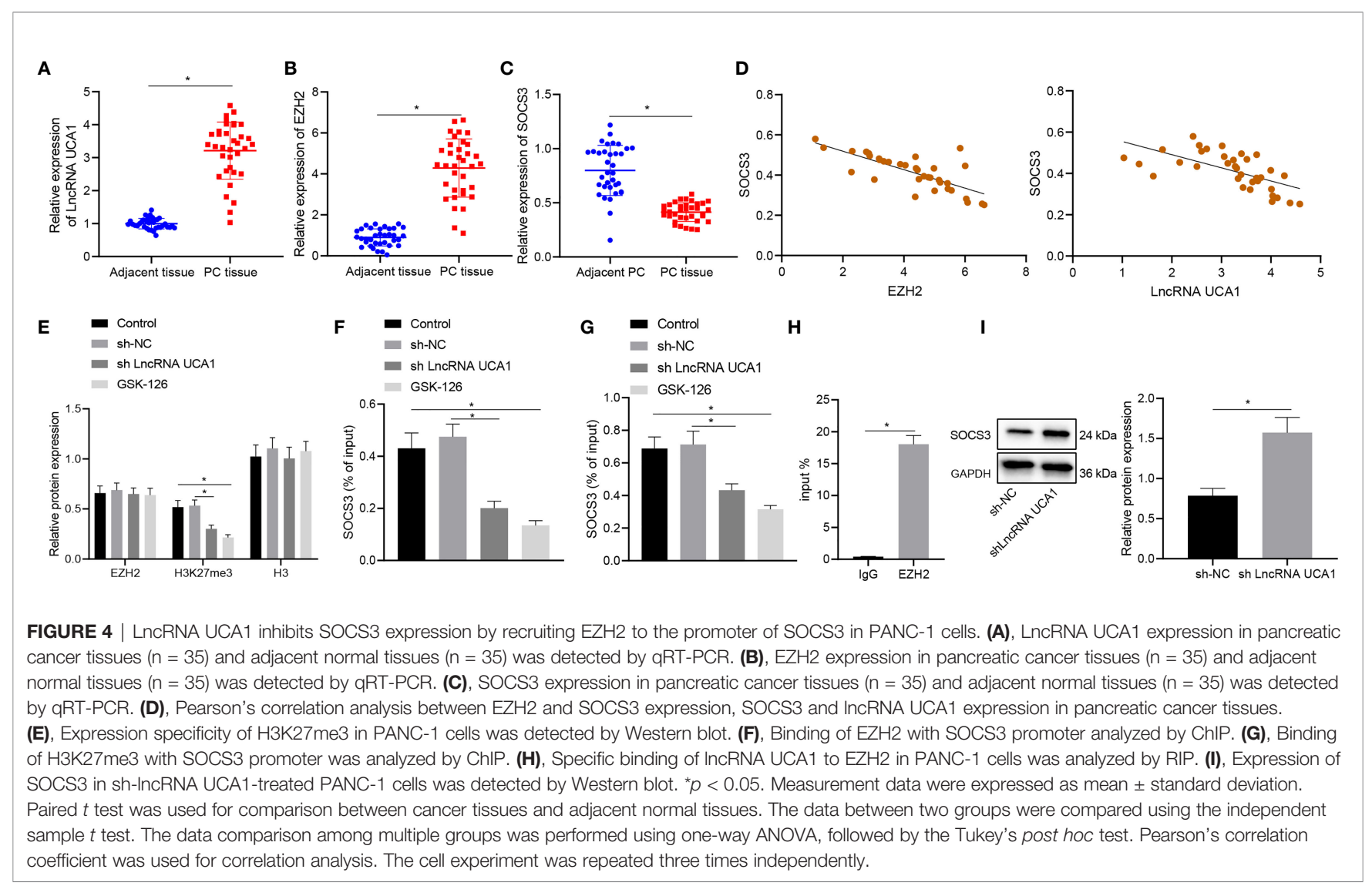

\section{LncRNA UCA1 Delivered by HPSC-EXO Promotes Gem Resistance and Tumorigenesis of Pancreatic Cancer Cells by Regulating the EZH2/SOCS3 Axis In Vitro and In Vivo}

Finally, we proceeded to determine whether HPSC-EXO affects the chemotherapy resistance of pancreatic cancer through the lncRNA UCA1/EZH2/SOCS3 axis. The results of qRT-PCR and Western blot showed that the expression of IncRNA UCA1 and SOCS3 was reduced in PANC-1 cells following lncRNA UCA1 knockdown while further SOCS3 overexpression enhanced the expression of SOCS3 (Figures 5A, B).

Moreover, the results of MTT assay (Figure $5 \mathrm{C}$ ) revealed a reduction in the viability of PANC-1 cells upon knockdown of lncRNA UCA1, while an enhancement was noted in the presence of both IncRNA UCA1 knockdown and SOCS3 overexpression. As depicted in Figures 5B, D, E, lncRNA UCA1 knockdown augmented cell apoptosis and increased the expression of cleaved caspase 3, the effect of which was abolished by further SOCS3 overexpression. Furthermore, the results of Transwell assay exhibited that lncRNA UCA1 knockdown resulted in inhibition in the migration and invasion of PANC-1 cells, which was negated by additional SOCS3 overexpression (Figures 5F, G). MTT assay data (Figure 5H) showed that PANC-1 cells treated with shlncRNA UCA1 + oe-SOCS3 had reduced sensitivity to Gem than those with sh-lncRNA UCA1 alone.
The results of in vivo experiments (Figures 5I-K) further showed that treatment with Gem reduced the tumor volume and weight of mice with lncRNA UCA1 knockdown as well as decreasing the positive expression rate of Ki67 in the tumor tissues. However, opposite results were noted in the presence of sh-lncRNA UCA1 + oe-SOCS3. The above results indicate that lncRNA UCA1 regulates the EZH2/SOCS axis to promote the Gem resistance and tumorigenesis of pancreatic cancer cells.

\section{DISCUSSION}

As a typical feature of most tumors, especially solid tumors, hypoxia microenvironment induces adaptive regulation of tumor and stromal cells, thus promoting the development and invasiveness of tumors (17). Tumor cells have been shown to release more exosomes in hypoxia microenvironment, which are involved in angiogenesis regulation (18). In the present study, we found that the hypoxic tumor microenvironment could induce activation of PSCs and enhance the secretion of exosomes from PSCs. The high expression of exosomal lncRNA UCA1 promoted the malignant phenotypes, and augmented the Gem resistance of pancreatic cancer.

Notably, the expression of IncRNA UCA1 is dysregulated in many malignant tumors, which affects tumor occurrence and development. Some studies have also reported that lncRNA 
A

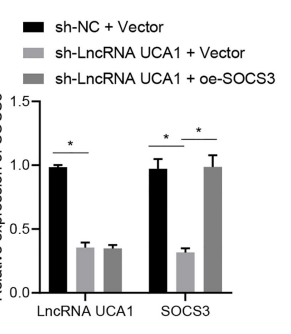

E
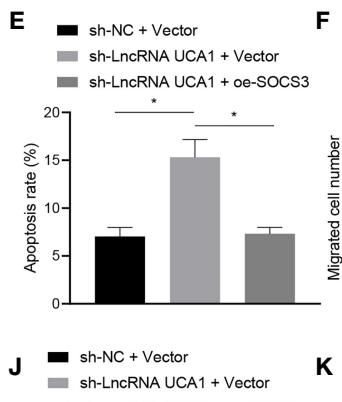

sh-LncRNA UCA1 + oe-SOCS3

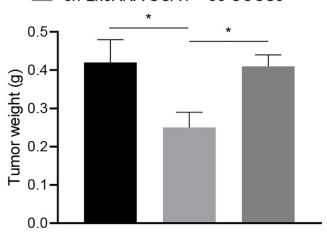

B
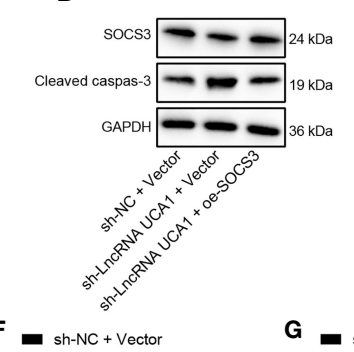

- sh-LncRNA UCA1 + Vector

- sh-LncRNA UCA1 + oe-SOCS3

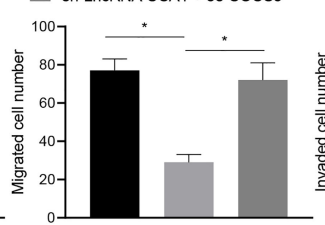

$G$

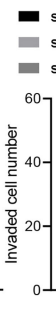

$40-$

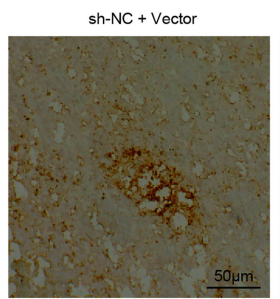

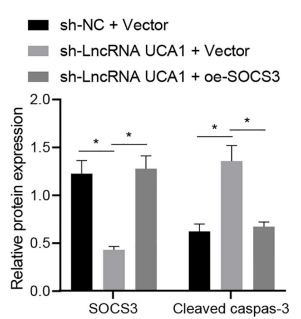

C

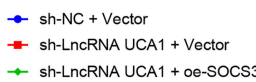

$\rightarrow$ sh-LncRNA UCA1 + oe-SOCS3

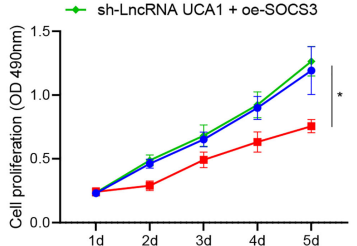

D

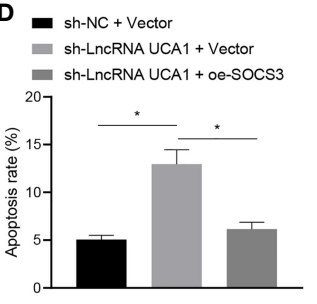

H

$\rightarrow$ sh-NC + Vector

* sh-LncRNA UCA1 + Vector

$\rightarrow$ sh-LncRNA UCA1 + oe-SOCS3

I

$\rightarrow$ sh-NC + Vector

* sh-LncRNA UCA1 + Vector

$\rightarrow$ sh-LncRNA UCA1 + oe-SOCS3

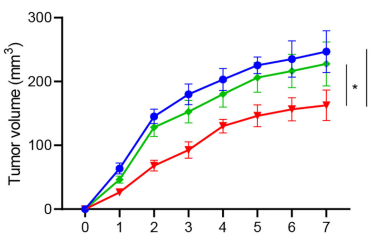

- sh-NC+ Vector

- sh-LncRNA UCA1 + Vector

sh-LncRNA UCA1 + oe-SOCS3
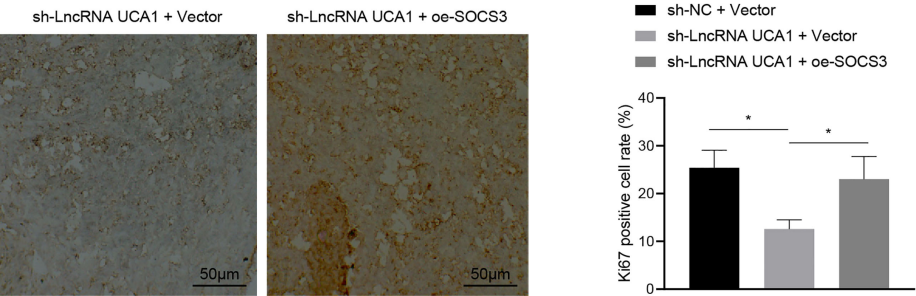

FIGURE 5 | LnCRNA UCA1 delivered by HPSC-EXO enhances Gem resistance and promotes tumorigenesis of pancreatic cancer by recruiting EZH2 and downregulating SOCS3 expression. (A), Expression of IncRNA UCA1 and SOCS3 in PANC-1 cells treated with sh-IncRNA UCA1 or combined with oe-SOCS3 was detected by qRT-PCR. (B), Expression of cleaved caspase 3 and SOCS3 in PANC-1 cells treated with sh-IncRNA UCA1 or combined with oe-SOCS3 was detected by Western blot. (C), Viability of PANC-1 cells treated with sh-IncRNA UCA1 or combined with oe-SOCS3 was detected by MTT assay. (D), Apoptosis of PANC-1 cells treated with sh-IncRNA UCA1 or combined with oe-SOCS3 was detected by flow cytometry. (E), Apoptosis of PANC-1 cells treated with sh-IncRNA UCA1 or combined with oe-SOCS3 was detected by TUNEL staining. (F), Migration of PANC-1 cells treated with sh-IncRNA UCA1 or combined with oe-SOCS3 was detected by Transwell assay. (G), Invasion of PANC-1 cells treated with sh-IncRNA UCA1 or combined with oe-SOCS3 was detected by Transwell assay. (H), Gem sensitivity of PANC-1 cells treated with sh-IncRNA UCA1 or combined with oe-SOCS3 analyzed by MTT method. (I), Tumor volume of mice treated with sh-IncRNA UCA1 or combined with oe-SOCS3 after injection of Gem. (J), Tumor weight of mice treated with sh-IncRNA UCA1 or combined with oe-SOCS3 after injection of Gem. (K), The positive expression rate of Ki67 in tumor tissues of mice treated with sh-IncRNA UCA1 or combined with oe-SOCS3 after injection of Gem was detected by immunohistochemistry. ${ }^{\star} P<0.05$. Measurement data were expressed as mean \pm standard deviation. The data comparison among multiple groups was performed using one-way ANOVA, followed by the Tukey's post hoc test. The data at different time points were compared by repeated measures ANOVA, followed by Bonferroni's post hoc test. The cell experiment was repeated three times. $n=8$ for mice following each treatment.

UCA1 is highly expressed in pancreatic cancer tissues and plays a carcinogenic role in tumor proliferation and metastasis $(19,20)$. In addition, lncRNA UCA1 expression was found not only to be increased in hypoxic pancreatic cancer cells, but also enriched in exosomes derived from hypoxic pancreatic cancer cells, and it was established that IncRNA UCA1 knockout restricted angiogenesis and tumor growth (7). These results suggested that hypoxic pancreatic cancer cell-derived exosome-mediated angiogenesis was dependent on IncRNA UCA1.

It is well acknowledged that lncRNAs function via various mechanisms, which are mainly based on the cellular location. We also showed that knockdown of lncRNA UCA1 inhibited tumorigenesis in mice xenografting with pancreatic cancer cells. EZH2 overexpression occurred in pancreatic cancer and it was positively correlated with lncRNA UCA1 expression. Previous evidence showed that IncRNA UCA1 was upregulated in hepatocellular carcinoma, and EZH2 showed a negative correlation with lncRNA UCA1 level (12). EZH2 is a lysine methyltransferase, which can synergize with lncRNA UCA1. LncRNA UCA1 was up-regulated in non-small-cell lung cancer tissues and gefitinib resistant cells, indicating that lncRNA UCA1 played a special role in gefitinib resistance, and lncRNA UCA1 knockout can increase the sensitivity to gefitinib by inhibiting cell proliferative capacities by interacting with EZH2 to epigenetically reduce CDKN1A expression (21). miR124 delivered by bone marrow mesenchymal stromal cellderived exosomes can directly inhibit the expression of EZH2, and consequently suppress the malignant phenotypes of pancreatic tumor cells, and sensitizes pancreatic cancer cells to chemotherapy in vitro and in vivo (22). In addition, tumorderived exosomal LINC01133 can interact with EZH2 and then promotes $\mathrm{H} 3 \mathrm{~K} 27$ trimethylation, contributing to pancreatic ductal adenocarcinoma tumor growth and epithelialmesenchymal transition in vivo (23). 
Further, we observed that SOCS3 underexpression occurred in pancreatic cancer and it was negatively correlated with EZH2. In addition, IncRNA UCA1 was involved in EZH2 mediated epigenetic inhibition of SOCS3 in PANC-1 cells by binding to EZH2. It was previously found that the expression of SOCS3 in hepatocellular carcinoma tissues and cell lines was negatively correlated with EZH2 and depended on its promoter methylation status through TCGA hepatocellular carcinoma data analysis. The promoter recruitment of SOCS3 gene by CTCF dependent EZH2 may be involved in the epigenetic silencing of SOCS3 and the regulation of its gene expression (13). It was shown in in vivo and in vitro experiments of a previous study, upregulated GAS5 promoted the expression of SOCS3, thus inhibiting the growth, metastasis, and Gem resistance of pancreatic cancer (14). Corroborating previous documentation, our results confirmed through gain- and lossof function assays that lncRNA UCA1 inhibited the expression of
SOCS3, thereby enhancing the malignant phenotypes, tumorigenesis and Gem resistance of pancreatic cancer cells.

\section{CONCLUSION}

In conclusion, the exosomes secreted by hypoxia-activated PSCs could deliver lncRNA UCA1 to pancreatic cancer cells. By recruiting EZH2, lncRNA UCA1 regulates the methylation of SOCS3 protein, reduces the expression of SOCS3, and promotes the Gem resistance of pancreatic cancer (Figure 6). Thus, our study provides interesting targets for pancreatic cancer prevention and treatment. Future studies are warranted to understand the mechanistic link between our findings and potential association with tumor reactive oxygen species, and the ensuing instability of tumor genome and formation of new blood vessels.

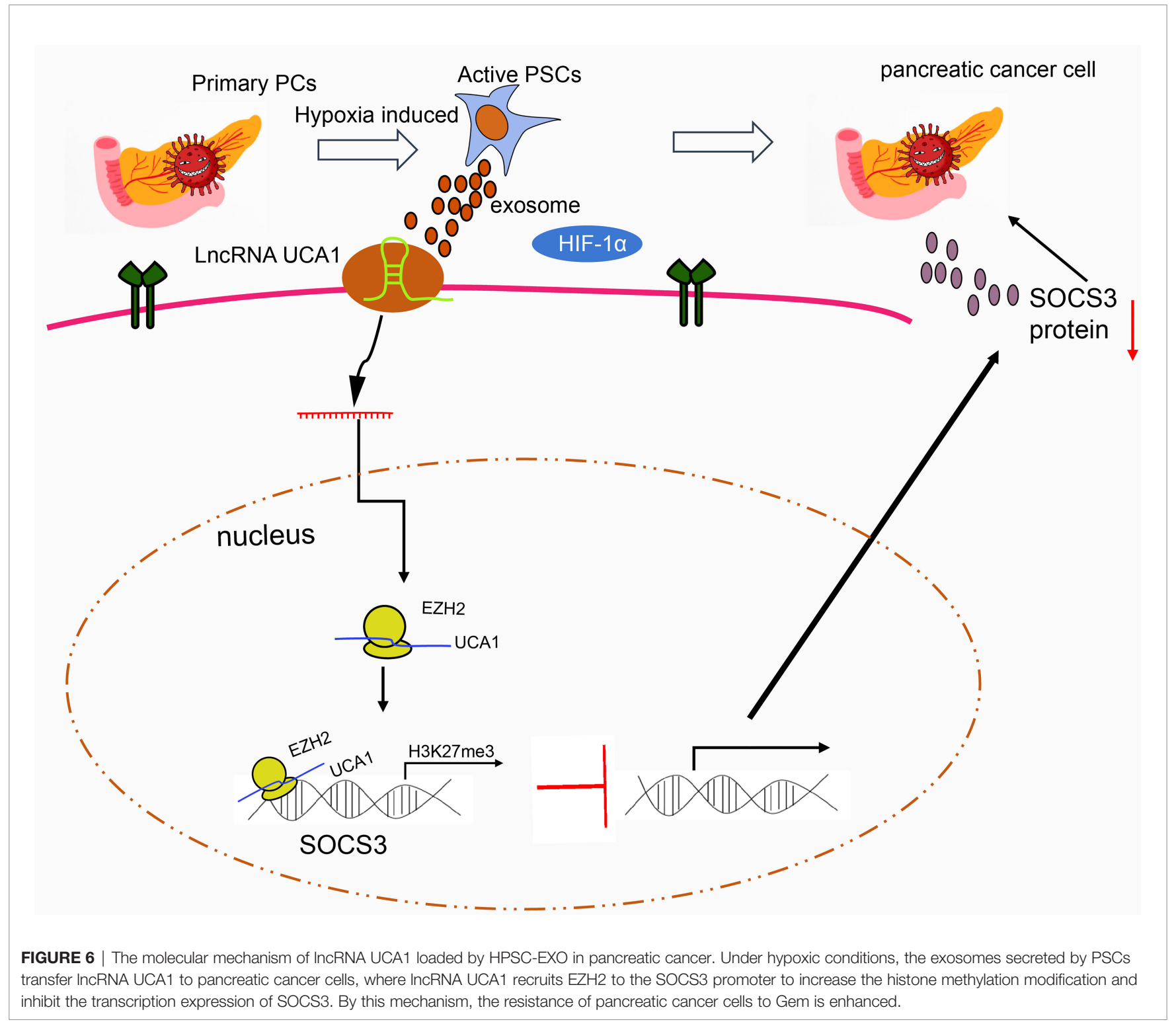




\section{DATA AVAILABILITY STATEMENT}

The original contributions presented in the study are included in the article/Supplementary Material. Further inquiries can be directed to the corresponding author.

\section{ETHICS STATEMENT}

The studies involving human participants were reviewed and approved by the Ethics Committee of Shengjing Hospital of China Medical University. The patients/participants provided their written informed consent to participate in this study. The animal study was reviewed and approved by the Animal Ethics Committee of Shengjing Hospital of China Medical University.

\section{AUTHOR CONTRIBUTIONS}

YC and ZL designed the study and drafted the paper. HX and ZL were involved in data collection. YC and HX performed the

\section{REFERENCES}

1. Moutinho-Ribeiro P, Macedo G, Melo SA. Pancreatic Cancer Diagnosis and Management: Has the Time Come to Prick the Bubble? Front Endocrinol (Lausanne) (2018) 9:779. doi: 10.3389/fendo.2018.00779

2. Pothula SP, Xu Z, Goldstein D, Pirola RC, Wilson JS, Apte MV. Key Role of Pancreatic Stellate Cells in Pancreatic Cancer. Cancer Lett (2016) 381(1):194200. doi: 10.1016/j.canlet.2015.10.035

3. Yu F, Dong B, Dong P, He Y, Zheng J, Xu P. Hypoxia Induces the Activation of Hepatic Stellate Cells Through the PVT1-miR-152-ATG14 Signaling Pathway. Mol Cell Biochem (2020) 465(1-2):115-23. doi: 10.1007/s11010019-03672-y

4. Zhao W, Ajani JA, Sushovan G, Ochi N, Hwang R, Hafley M, et al. Galectin-3 Mediates Tumor Cell-Stroma Interactions by Activating Pancreatic Stellate Cells to Produce Cytokines via Integrin Signaling. Gastroenterology (2018) 154(5):1524-37.e6. doi: 10.1053/j.gastro.2017.12.014

5. Principe DR, Narbutis M, Kumar S, Park A, Viswakarma N, Dorman MJ, et al. Long-Term Gemcitabine Treatment Reshapes the Pancreatic Tumor Microenvironment and Sensitizes Murine Carcinoma to Combination Immunotherapy. Cancer Res (2020) 80(15):3101-15. doi: 10.1158/00085472.CAN-19-2959

6. Amrutkar M, Vethe NT, Verbeke CS, Aasrum M, Finstadsveen AV, Santha P, et al. Differential Gemcitabine Sensitivity in Primary Human Pancreatic Cancer Cells and Paired Stellate Cells Is Driven by Heterogenous Drug Uptake and Processing. Cancers (Basel) (2020) 12(12):3628. doi: 10.3390/ cancers 12123628

7. Guo Z, Wang X, Yang Y, Chen W, Zhang K, Teng B, et al. Hypoxic TumorDerived Exosomal Long Noncoding RNA UCA1 Promotes Angiogenesis via miR-96-5p/AMOTL2 in Pancreatic Cancer. Mol Ther Nucleic Acids (2020) 22:179-95. doi: 10.1016/j.omtn.2020.08.021

8. Pitt JM, Kroemer G, Zitvogel L. Extracellular Vesicles: Masters of Intercellular Communication and Potential Clinical Interventions. J Clin Invest (2016) 126 (4):1139-43. doi: 10.1172/JCI87316

9. van Niel G, D’Angelo G, Raposo G. Shedding Light on the Cell Biology of Extracellular Vesicles. Nat Rev Mol Cell Biol (2018) 19(4):213-28. doi: 10.1038/nrm.2017.125

10. Xue M, Chen W, Xiang A, Wang R, Chen H, Pan J, et al. Hypoxic Exosomes Facilitate Bladder Tumor Growth and Development Through Transferring Long Non-Coding RNA-Ucal. Mol Cancer (2017) 16(1):143. doi: 10.1186/ s12943-017-0714-8 statistical analysis and preparation of figures. All authors contributed to the article and approved the submitted version.

\section{FUNDING}

This study was supported by General projects of the National Natural Science Foundation of China (8187071004) and the 345 Talent Project.

\section{SUPPLEMENTARY MATERIAL}

The Supplementary Material for this article can be found online at: https://www.frontiersin.org/articles/10.3389/fonc.2021.671082/ full\#supplementary-material

Supplementary Figure 1 | Gem resistance assay. (A), Dose-response curve of Gem in the parental and GemMIA-R3 cell lines. Data represent the mean \pm standard error of at least three independent experiments. (B), Cell growth inhibition data (GI50) of the parental and three Gem-resistant MIA PaCa-2 cell lines. Unit: nM. The cell experiment was repeated three times.

11. Xie W, Chu M, Song G, Zuo Z, Han Z, Chen C, et al. Emerging Roles of Long Noncoding RNAs in Chemoresistance of Pancreatic Cancer. Semin Cancer Biol (2020) 15:S1044-579X(20)30222-4. doi: 10.1016/j.semcancer.2020.11.004

12. Hu JJ, Song W, Zhang SD, Shen XH, Qiu XM, Wu HZ, et al. HBX-Upregulated lncRNA UCA1 Promotes Cell Growth and Tumorigenesis by Recruiting EZH2 and Repressing p27Kip1/CDK2 Signaling. Sci Rep (2016) 6:23521. doi: 10.1038/srep23521

13. Wei L, Liu Q, Huang Y, Liu Z, Zhao R, Li B, et al. Knockdown of CTCF Reduces the Binding of EZH2 and Affects the Methylation of the SOCS3 Promoter in Hepatocellular Carcinoma. Int J Biochem Cell Biol (2020) 120:105685. doi: 10.1016/j.biocel.2020.105685

14. Liu B, Wu S, Ma J, Yan S, Xiao Z, Wan L, et al. lncRNA GAS5 Reverses EMT and Tumor Stem Cell-Mediated Gemcitabine Resistance and Metastasis by Targeting miR-221/SOCS3 in Pancreatic Cancer. Mol Ther Nucleic Acids (2018) 13:472-82. doi: 10.1016/j.omtn.2018.09.026

15. Hermreck AS, Thomas CY, Friesen SR. Importance of Pathologic Staging in the Surgical Management of Adenocarcinoma of the Exocrine Pancreas. Am J Surg (1974) 127(6):653-7. doi: 10.1016/0002-9610(74)90341-9

16. Xiao Y, Qin T, Sun L, Qian W, Li J, Duan W, et al. Resveratrol Ameliorates the Malignant Progression of Pancreatic Cancer by Inhibiting Hypoxia-Induced Pancreatic Stellate Cell Activation. Cell Transplant (2020) 29:963689720929987. doi: 10.1177/0963689720929987

17. Rankin EB, Giaccia AJ. Hypoxic Control of Metastasis. Science (2016) 352 (6282):175-80. doi: 10.1126/science.aaf4405

18. Kucharzewska P, Christianson HC, Welch JE, Svensson KJ, Fredlund E, Ringner $\mathrm{M}$, et al. Exosomes Reflect the Hypoxic Status of Glioma Cells and Mediate Hypoxia-Dependent Activation of Vascular Cells During Tumor Development. Proc Natl Acad Sci USA (2013) 110(18):7312-7. doi: 10.1073/pnas.1220998110

19. Yu X, Lin Y, Sui W, Zou Y, Lv Z. Analysis of Distinct Long Noncoding RNA Transcriptional Fingerprints in Pancreatic Ductal Adenocarcinoma. Cancer Med (2017) 6(3):673-80. doi: 10.1002/cam4.1027

20. Zhang X, Gao F, Zhou L, Wang H, Shi G, Tan X. UCA1 Regulates the Growth and Metastasis of Pancreatic Cancer by Sponging miR-135a. Oncol Res (2017) 25(9):1529-41. doi: 10.3727/096504017X14888987683152

21. Xu T, Yan S, Wang M, Jiang L, Ma P, Lu B, et al. LncRNA UCA1 Induces Acquired Resistance to Gefitinib by Epigenetically Silencing CDKN1A Expression in Non-Small-Cell Lung Cancer. Front Oncol (2020) 10:656. doi: $10.3389 /$ fonc. 2020.00656

22. Xu Y, Liu N, Wei Y, Zhou D, Lin R, Wang X, et al. Anticancer Effects of miR-124 Delivered by BM-MSC Derived Exosomes on Cell Proliferation, 
Epithelial Mesenchymal Transition, and Chemotherapy Sensitivity of Pancreatic Cancer Cells. Aging (Albany NY) (2020) 12(19):19660-76. doi: 10.18632/aging.103997

23. Liu $\mathrm{Y}$, Tang T, Yang $\mathrm{X}$, Qin $\mathrm{P}$, Wang $\mathrm{P}$, Zhang $\mathrm{H}$, et al. Tumor-Derived Exosomal Long Noncoding RNA LINC01133, Regulated by Periostin, Contributes to Pancreatic Ductal Adenocarcinoma Epithelial-Mesenchymal Transition Through the Wnt/beta-Catenin Pathway by Silencing AXIN2. Oncogene (2021) 40(17):3164-79. doi: 10.1038/s41388-021-01762-0

Conflict of Interest: The authors declare that the research was conducted in the absence of any commercial or financial relationships that could be construed as a potential conflict of interest.
Publisher's Note: All claims expressed in this article are solely those of the authors and do not necessarily represent those of their affiliated organizations, or those of the publisher, the editors and the reviewers. Any product that may be evaluated in this article, or claim that may be made by its manufacturer, is not guaranteed or endorsed by the publisher.

Copyright $\odot 2021$ Chi, Xin and Liu. This is an open-access article distributed under the terms of the Creative Commons Attribution License (CC BY). The use, distribution or reproduction in other forums is permitted, provided the original author(s) and the copyright owner(s) are credited and that the original publication in this journal is cited, in accordance with accepted academic practice. No use, distribution or reproduction is permitted which does not comply with these terms. 